\title{
On-Line Determination of Cyclophosphamide in Blood Samples Utilizing Microextraction by Packed Sorbent and Liquid Chromatography Tandem Mass Spectrometry (MEPS-LC-MS/MS)
}

\author{
Mohamed Kamel ${ }^{1}$, Rana Said ${ }^{2}$, Aziza Elbeqqali $^{1}$, Fatma Bassyouni $^{3}$ and \\ Mohamed Abdel-Rehim ${ }^{*}, 1,3,4$
}

\author{
${ }^{I}$ Department of Chemistry \& Biomedical Sciences, Faculty of Technology and Science, Karlstad University, SE-651 88 \\ Karlstad, Sweden \\ ${ }^{2}$ Karolinska Institute, Karolinska University Hospital, Stockholm, Sweden \\ ${ }^{3}$ Department of Pharmaceutical Research, Center of Excellence for Advanced Sciences and Department of Natural and \\ Microbial Products, National Research Centre, Dokki, Cairo, Egypt \\ ${ }^{4}$ Department of Clinical Pharmacology \& DMPK, AstraZeneca, R\&D, Södertälje, 15185 Södertälje, Sweden
}

\begin{abstract}
Microextraction by packed sorbent (MEPS) was used to handle whole mice blood samples. MEPS was used as an online rapid sample-preparation method, followed by liquid chromatography with tandem mass spectrometry (LCMS/MS). Cyclophosphamide in mice blood was used as a model compound. The new method reduced the handling time and the cost and could handle small volumes of whole blood samples $(20 \mu \mathrm{L})$. The lower limit of quantification (LLOQ) was $0.1 \mu \mathrm{g} / \mathrm{mL}$. The accuracy of the quality-control (QC) samples ranged from 107 to $110 \%$. The within-day variation was within the range $2.0-7.0 \%$ (C.V.\%) while the between-day variation was between 4.0 and $6.0 \%$ (C.V.\%). The calibration curve in whole blood was constructed within the concentration range $0.1-100 \mu \mathrm{g} / \mathrm{mL}$. The coefficients of determination $\left(\mathrm{R}^{2}\right)$ were $>0.99(\mathrm{n}=3)$. The present method is rapid, reliable and accurate and may be used for therapeutic drug monitoring of cyclophosphamide in whole blood. The method was applied for preclinical study of cyclophosphamide in mice.
\end{abstract}

Keywords: Whole blood, microextraction by packed sorbent, cyclophosphamide, LC-MS/MS.

\section{INTRODUCTION}

The sample preparation is the first and important stage in chromatographic bioanalysis. In cases when the analytes are present in a complex matrix, e.g. blood or urine, the sample preparation is of crucial importance for the analysis. Today the extraction of drugs and metabolites from biological samples has been changed in many ways. There is an everincreasing interest in the direct analysis of whole blood samples. The demands on a sample preparation procedure are: it must be highly repeatable, with a high recovery of the target analytes. In addition, direct analysis of blood samples should reduce the sample handling time and involve a minimum number of working steps. Micro extraction by packed sorbent (MEPS) is a miniaturized solid phase extraction (SPE) cartridge integrated into a gas tight syringe that can be connected online to GC or LC without any modification. The MEPS consists of two parts, the MEPS Syringe (100, 250 $\mu \mathrm{L}$ ) and the MEPS Barrel Insert and Needle Assembly (BIN) that contains about $2 \mathrm{mg}$ of sorbent. The dead volume is about $7 \mu \mathrm{L}$. In MEPS the volume of solvent used to elute the analytes from the extraction process is of a suitable order of

*Address correspondence to this author at the Department of Chemistry \& Biomedical Sciences, Faculty of Technology and Science, Karlstad University, SE-651 88 Karlstad, Sweden;

E-mail: Mohamed.Abdel-Rehim@astrazeneca.com magnitude to be injected directly into a LC or GC system. Effectively, MEPS is an LC column in a syringe. The bed dimensions are scaled from the conventional SPE bed and so MEPS can be adapted into most existing SPE methods simply by scaling the reagents and sample volumes from the conventional device to the MEPS. The technique has been employed to extract a wide range of analytes in many areas [1-6].

The aim of the present study was to investigate MEPS as sample preparation method to handle small whole blood samples $(\leq 20 \mu \mathrm{L})$. Cyclophosphamide in whole mice blood, from a preclinical study, was used as a model substance. Cyclophosphamide is one of the most used anti-cancer drugs $[7,8]$. It is used in the treatment of malignant diseases such as leukemia, lymphoma and solid tumors.

\section{MATERIAL AND METHODS}

\subsection{Chemicals and Materials}

Cyclophosphamide was purchased from Sigma Chemical Company (St. Louis, MO, USA). Internal standard was ${ }^{2} \mathrm{H}_{3}-$ lidocaine and obtained from Department of Medicinal Chemistry, AstraZeneca, Södertälje, Sweden. Acetonitrile, methanol, formic acid and ammonium hydroxide were obtained from Merck (Darmstadt, Germany). All chemicals were of analytical grade. The Milli-Q water used was ob- 
tained from Reagent Grade Milli-Q Plus water purification system (Millipore Corporation, Bedford, USA).

\subsection{Apparatus}

MEPS-LC-MS/MS: The MEPS syringe (Fig. 1), $(250 \mu \mathrm{L}$ syringe, C2 sorbent) was obtained from SGE (Melbourne, Australia).

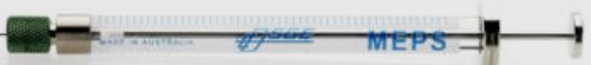

Fig. (1). MEPS syringe from SGE.

The liquid chromatography (LC) instrument included two pumps, Shimadzu VP10DA (Kyoto, Japan), and an autosampler, CTC-Pal, (CTC Analytics AG, Zwingen, Switzerland). An Ace $(100 \times 2.1 \mathrm{~mm}, \mathrm{C} 18,3 \mathrm{~mm})$ column obtained from Advanced Chromatography Technologies (Aberdeen, Scotland) was used as analytical column. A guard column; Optiguard $(\mathrm{C} 8,10 \times 1 \mathrm{~mm})$, was obtained from Optimize Technologies (Oregon City, OR, USA). Gradient HPLC was used. Mobile phase A was $0.1 \%$ formic acid in water and acetonitrile 90:10 (v/v) and mobile phase B contained $0.1 \%$ formic acid in water and acetonitrile 20:80 (v/v). The gradient started from $1.0 \%$ of phase B up to $50 \%$ from 1 to $4 \mathrm{~min}$ and then from 4 to $6 \mathrm{~min}$ to $90 \%$ of phase $\mathrm{B}$ and at $6.5 \mathrm{~min}$ phase B was set at $1.0 \%$ again. The flow rate was 150 $\mu \mathrm{L} / \mathrm{min}$ and sample volume was $40 \mu \mathrm{L}$.

All experiments were conducted using a triple quadrupole mass spectrometric instrument, Micromass QuatroUltima (Waters Corporation, Manchester, UK) equipped with a Z-electrospray interface operated in positive ion mode. The scan mode was multiple reaction monitoring (MRM) using precursor ion at (M+1) $(\mathrm{m} / \mathrm{z}: 261$ and 237.9) and after collisional dissociation the product ions $\mathrm{m} / \mathrm{z}: 140$ and 86 were used for quantification of cyclophosphamide and the internal standard, respectively. Fig. (2) shows a full scan ESI-MS of cyclophosphamide and the CID-MS/MS of the protonated molecule $[\mathrm{M}+\mathrm{H}]^{+}$at $m / z 261$.

The optimum mass spectrometric parameter settings were: capillary voltage at $3 \mathrm{kV}$, cone voltage at $27 \mathrm{~V}$, extractor at $5 \mathrm{~V}, \mathrm{RF}$ lens at $0.2 \mathrm{~V}$, source block and desolvation temperatures at $150{ }^{\circ} \mathrm{C}$ and $300{ }^{\circ} \mathrm{C}$, respectively. Nitrogen was used both as drying $\left(400 \mathrm{~L} \mathrm{~h}^{-1}\right)$, and nebulizing gases $\left(20 \mathrm{~L} \mathrm{~h}^{-1}\right)$; the vacuum was $2 \times 10^{-5} \mathrm{mbar}$ in the mass analyzer and $2 \times 10^{-3}$ mbar in the collision cell. Argon was used as collision gas and collision energy was $22 \mathrm{eV}$. The gases were from ScanGas (Stockholm, Sweden). The data were collected and processed using MassLynx version 4.0, and all calculations were based on peak area ratios.

Two mice groups were administered cylophosphamide intraperitoneally at a dose of $100 \mathrm{mg} / \mathrm{kg}$ and blood samples were collected $0.25,0.5,1,2,3,4,5$ and 6 hours. The blood samples were collected serially from the tail vein and about $20 \mu \mathrm{L}$ of blood was collected using syringe containing $80 \mu \mathrm{L}$ $0.5 \mathrm{M}$ EDTA. All samples were stored in a freezer set at $20^{\circ} \mathrm{C}$ until its analysis.

\subsection{Sample Preparation}

The calibration curve in whole blood was obtained in the concentration range $0.1-100 \mu \mathrm{g} / \mathrm{mL}(0.1,0.5,1.0,10,25,50$ and $100 \mathrm{mg} / \mathrm{mL}$ ). Quality control (QC) samples concentrations were $0.4,40$ and $80 \mu \mathrm{g} / \mathrm{mL}$. Aliquots of $20 \mu \mathrm{L}$ of blood samples, standards and QC samples, were diluted with water $(1: 25)$ and $20 \mu \mathrm{L}$ of IS $(40 \mu \mathrm{g} / \mathrm{mL})$ was added before MEPS extraction. The MEPS sorbent was manually conditioned with $50 \mu \mathrm{L}$ methanol followed by $50 \mu \mathrm{L}$ of water. After that, the syringe was connected to the autosampler and the spiked blood was withdrawn into the syringe by the autosampler. The sorbent was then washed by CTC-autosampler once with $100 \mu \mathrm{L}$ of water/methanol $(95: 5, \mathrm{v} / \mathrm{v})$ to remove proteins and other interferences. The analytes were then desorbed by $40 \mu \mathrm{L}$ methanol/water 90:10 (v/v) directly into the LC injector. Cleaning of the sorbent was carried out using $4 \times 250 \mu \mathrm{L}$ elution solution followed by $4 \times 250 \mu \mathrm{L}$ of the washing solution after every extraction. This step decreased memory effects, but also functioned as conditioning step before the next extraction. The same packing bed was used for about 40-100 extractions before it was discarded.
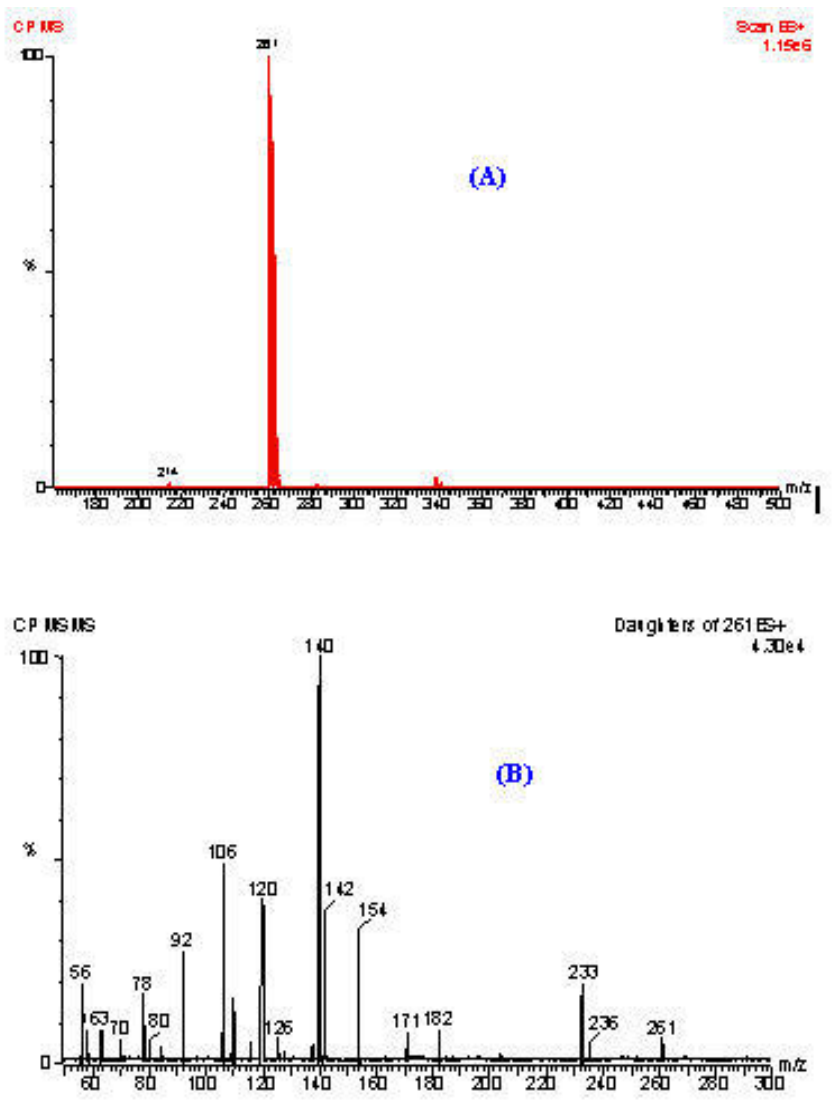

Fig. (2). Full scan ESI-MS of cyclophosphamide (A) and the CIDMS/MS of the protonated molecule $[\mathrm{M}+\mathrm{H}]^{+}$at $m / z 261$ (B).

\subsection{Validation}

Each calibration curve consisted of seven calibration points in mice blood covering from $0.1 \mu \mathrm{g} / \mathrm{mL}$ to $100 \mu \mathrm{g} / \mathrm{mL}$. Blank samples were run simultaneously. The peak area ratios for cyclophosphamide and internal standard were measured and a calibration curve without zero concentration was constructed. The calibration curves were described by the equation:

$$
y=a x^{2}+b x+c
$$

where $y$ is peak area ratio, $x$ the concentration, a curvature, $b$ the slope and $c$ the intercept. The calibration curves were 
quadric and the weight was $1 / x$. The accuracy and precision were calculated for the QC samples at three different assays.

Accuracy was defined as the degree of deviation of the determined value and the nominal value: [(measured valuenominal value) / nominal value] * 100. Precision (C.V. \%) was defined as the percentage of standard deviation of the observed values divided by their mean values: [(standard deviation) / mean value]* 100 .

\section{RESULTS AND DISCUSSION}

\subsection{Calibrations}

Linear calibration curves of blood samples spiked with cyclophosphamide standards were obtained in the range 0.1 $100 \mu \mathrm{g} / \mathrm{mL}$. The coefficients of determination $\left(\mathrm{R}^{2}\right)$ were $>$ $0.99(\mathrm{n}=3)$. Table 1 shows the back-calculated values of standard calibration samples in whole blood.

Table 1. Back-Calculated Values of the Calibration Points of the Mice Blood Samples Using MEPS-LC-MS/MS

\begin{tabular}{|c|c|c|}
\hline Conc. $\boldsymbol{\mu g} / \mathbf{m L}$ & Mean Conc. $(\mathrm{n}=\mathbf{6}) \boldsymbol{\mu g} / \mathbf{m L}$ & C.V. $\%$ \\
\hline \hline 0.1 & 0.104 & 8 \\
0.5 & 0.44 & 7 \\
1.0 & 1.2 & 6 \\
10 & 10.9 & 4 \\
25 & 23.2 & 5 \\
50 & 52.3 & 4 \\
100 & 99.5 & 3 \\
\hline
\end{tabular}

\subsection{Selectivity and Matrix Effect}

The method selectivity was defined as non-interference with the endogenous substances in the regions of interest. LC-MS/MS analysis of the blank blood samples showed no presence of an endogenous interference peak with the quantification of cyclophosphamide. Representative chroma-

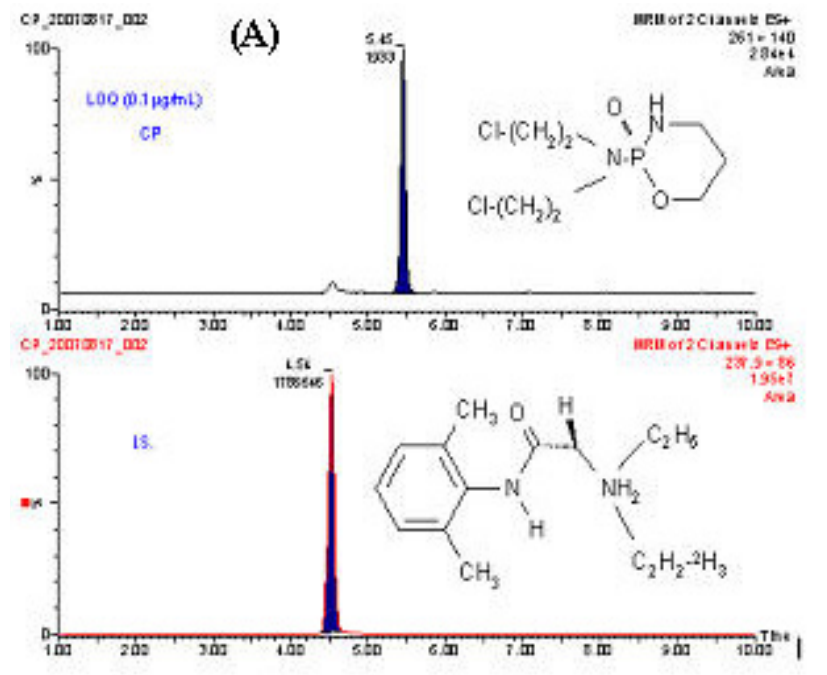

tograms of blank mice blood and spiked blood are presented in (Fig. 3).

In addition, the matrix effect on ionization was investigated by post-column continuous infusion of cyclophosphamide solution into the MS instrument and a pre-treated blank blood sample was injected into LC-MS. No ion suppression was observed at the retention time of cyclophosphamide.

\subsection{Accuracy and Precision}

The QC samples were prepared in mice blood. The accuracy is determined as the percentage difference from the nominal concentration value of QC samples $(n=12)$ at three different concentration levels. The between-day mean accuracy ranged from 107 to $109 \%$. The within-day variation of the precision (C.V.\%) was in the range $2.0-7.0 \%$. The between-day variation of the precision (C.V.\%) was in the range $4.0-6.0 \%$. The accuracy and precision results are summarized in Table 2.

Table 2. Within- and Between-Day Precision for Cyclophosphamide in Mice Blood Using MEPS-LC-MS/MS

\begin{tabular}{|c|c|c|c|}
\hline \multirow{2}{*}{ Concentration $\mu \mathrm{g} / \mathbf{m L}$} & Accuracy & \multicolumn{2}{|c|}{ Precision } \\
\cline { 2 - 4 } & $\begin{array}{c}\text { Accuracy \% } \\
(\mathbf{n}=\mathbf{1 2})\end{array}$ & $\begin{array}{c}\text { Within-Day } \\
(\mathbf{n = 6}) \\
(\mathbf{C . V . \% )}\end{array}$ & $\begin{array}{c}\text { Inter-Day } \\
(\mathbf{n}=\mathbf{1 2}) \\
(\mathbf{C . V . \% )}\end{array}$ \\
\hline \hline $80(\mathrm{QCH})$ & 109 & 2.0 & 6.0 \\
\hline $40(\mathrm{QCM})$ & 110 & 5.0 & 5.0 \\
\hline $0.4(\mathrm{QCL})$ & 107 & 7.0 & 4.0 \\
\hline $0.1(\mathrm{LLOQ})$ & 102 & 6.0 & 5.0 \\
\hline
\end{tabular}

\subsection{Extraction Recovery, Lower Limit of Quantification and Carry-Over}

The extraction recovery was determined by comparing the peak area of the extracted analyte from mice blood at two different concentrations (low- and high-quality control sam-

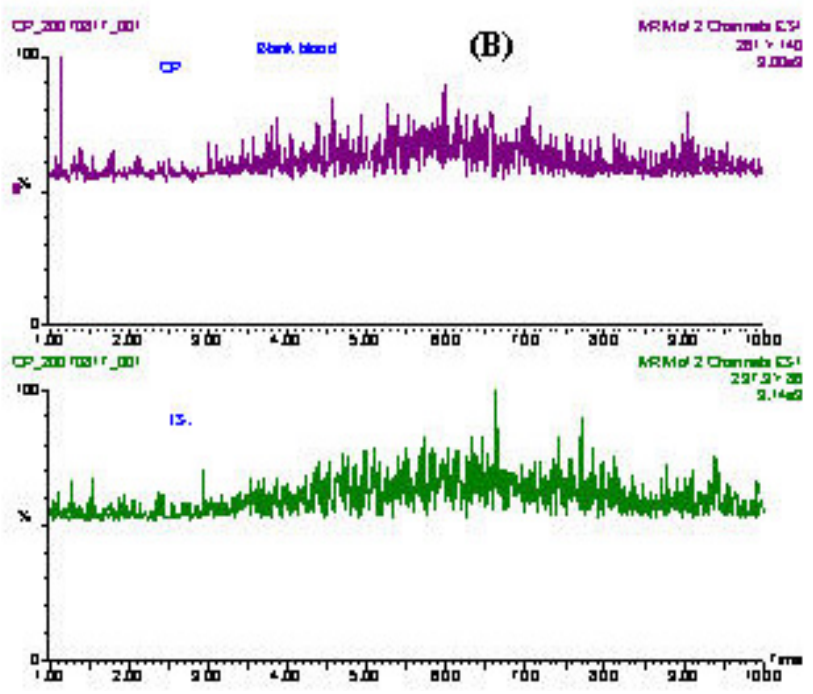

Fig. (3). Total ion chromatogram of the MRM analysis from: (A) mice blood spiked with cyclophosphamide 0.1 $\mu \mathrm{g} / \mathrm{mL}$ (LLOQ) and I.S.; (B) blank blood sample. 
ples) with the peak area obtained from standards prepared in mobile phase. The extraction recovery was over $90 \%$ when the sample was pumped four times $(4 \times 100 \mu \mathrm{L})$.

The lower limit of quantification (LLOQ) in mice blood was measured at $0.1 \mu \mathrm{g} / \mathrm{mL}$ (Fig. 3A).

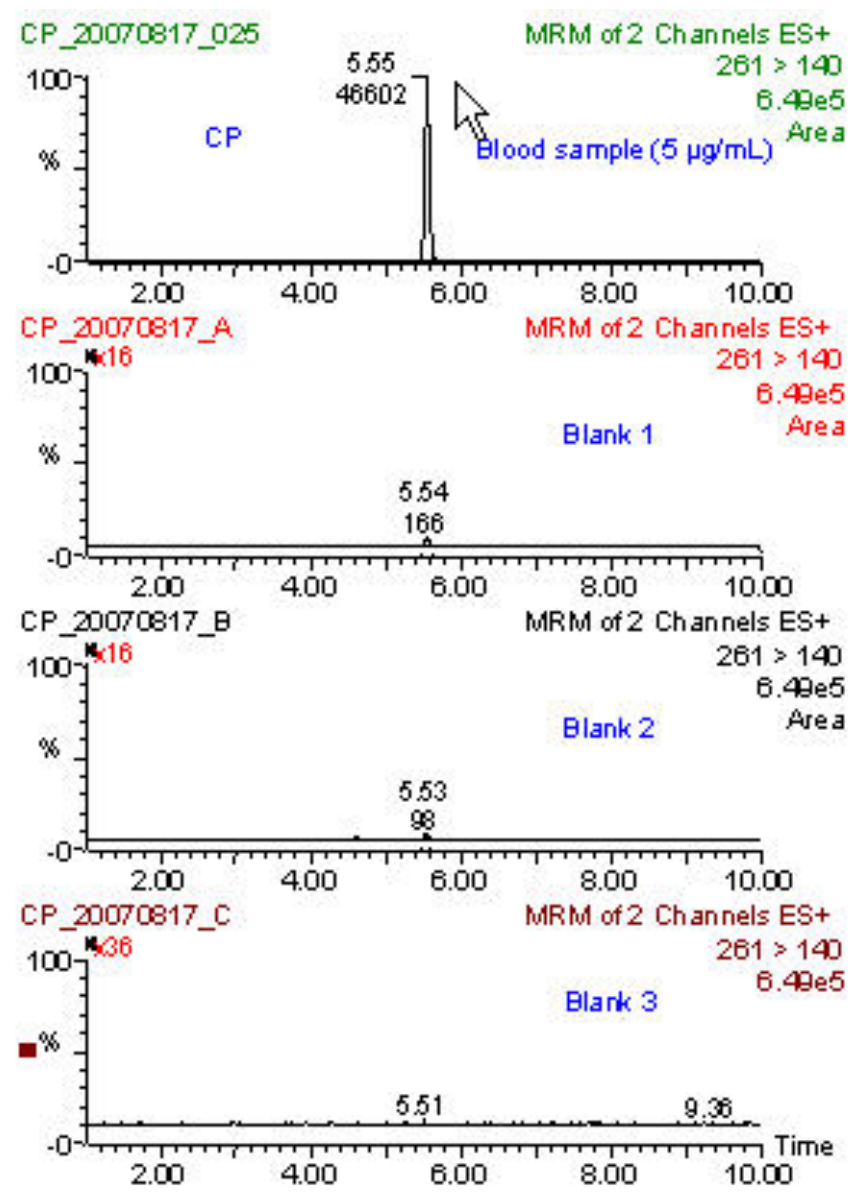

Fig. (4). Total ion chromatogram of the MRM analysis from (A) mice blood spiked with cyclophosphamide $5.0 \mu \mathrm{g} / \mathrm{mL}$ and three blank samples were injected after.

The precision (C.V.\%) for LLOQ was $5 \%(\mathrm{n}=12)$. The small amount of solid phase in the MEPS can be easily and effectively washed between samples to reduce the possibility of carry-over. After each injection, the syringe was washed five times with water and five times with methanol. The carry-over was about $0.4 \%$ after the first blank and after the third blank it was negligible (Fig. 4).

\subsection{Method Comparison}

Many previous methods used for the determination of the cyclophosphamide in plasma samples were performed using different sample preparation methods. In previous studies we used MEPS for extraction of drugs and metabolites from plasma and urine samples. In this paper we present, for first time, MEPS as a sample preparation method for the extraction of cyclophosphamide from whole blood samples. On the other hand it is important to mention that, further development of this assay is warranted to determine both cyclophosphamide and its active metabolites that may contribute to the clinical effects of the drug. Other research groups have adopted LC-MS techniques and LLE or SPE for the simultaneous determination of cyclophosphamide and its metabolites in plasma and tissue and have applied these assays in clinical and experimental trials. However, these methods [912] require long time work-up procedure. In the present study, we report a sample preparation method for whole blood, MEPS, that requires a few microliters of sample and extraction time for less than one minute.

The present method may play an important role to replace the old sample preparation methods and can be of a great advantage for therapeutic drug monitoring.

\subsection{Application of the Method}

In order to demonstrate the applicability of the proposed method, it was used to determine cyclophosphamide in mice blood samples from a preclinical study. A typical chromatogram of this type of samples appears in Fig. (5), where the concentration of cyclophosphamide found was $1.9 \mu \mathrm{g} / \mathrm{mL}$, resulting from a dose of $100 \mathrm{mg} / \mathrm{kg}$ administered.

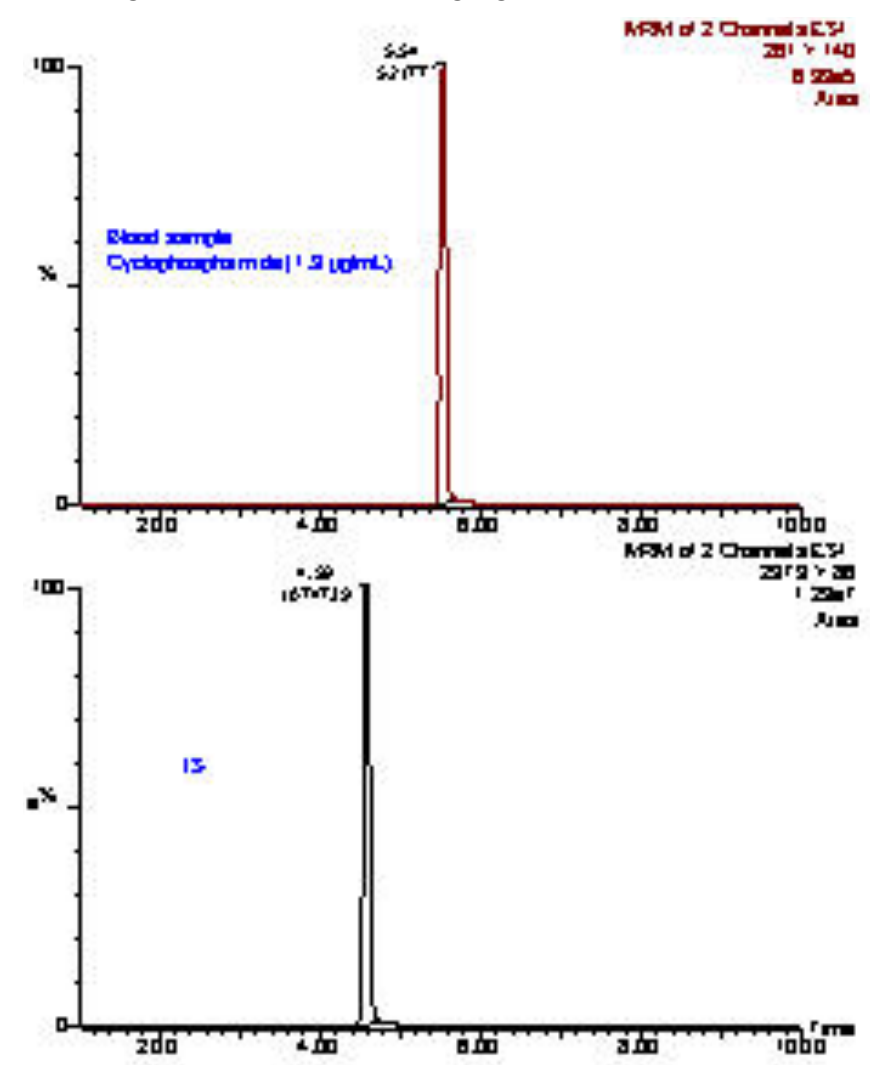

Fig. (5). Total ion chromatogram of the MRM analysis from a real mice blood sample of cyclophosphamide (planned dose: $100 \mathrm{mg} / \mathrm{kg}$, planned time: $1 \mathrm{hr}$ ).

\section{CONCLUSIONS}

This study has demonstrated that the combination of MEPS with LC-MS/MS can be a very useful tool for determination of drugs directly in a whole blood samples. The method is rapid and accurate for the determination of cyclophosphamide in whole blood. We demonstrated that carryover may be almost eliminated by washing the sorbent with elution and washing solutions. This approach to sample preparation is very promising for many reasons: 1) it is easy to use, 2) it is a fully automated online procedure, 3 ) it is rapid, and 4) the cost of analysis is minimal compared to 
conventional solid-phase extraction. Not only is the automation process with MEPS advantageous, but also the much smaller volumes of the samples and solvents provide other significant advantages such as the speed and the simplicity of the sample preparation process.

\section{REFERENCES}

[1] Abdel-Rehim M, Andersson A, Sandberg-Ställ M, et al. MEPS as a rapid sample preparation method to handle unstable compounds in a complex matrix: determination of AZD3409 in plasma samples utilizing MEPS-LC-MS-MS. J Chromatogr Sci 2008; 64: 518-23.

[2] El-Beqqali A, Abdel-Rehim M. Quantitative analysis of methadone in human urine samples by microextraction in packed syringe-gas chromatography-mass spectrometry (MEPS GC-MS). J Sep Sci 2007; 30: 2501-505.

[3] El-Beqqali A, Kussak A, Abdel-Rehim M. Determination of dopamine and serotonin in human urine samples utilizing microextraction online with liquid chromatography/electrospray tandem mass spectrometry. J liq Chromatogr Rel Technol 2007; 30: 575-86.

[4] Abdel-Rehim M, Askemark Y, Norsten-Höög C, Petterson J-K, Halldin M. Quantification of 4-OH-2,6-xylidine and its conjugates in human urine samples utilising microextraction in packed syringe on-line with liquid chromatography and electrospray tandem mass spectrometry (MEPS-LC-MS/MS). J Liq Chromatogr Rel Technol 2006; 29: 2413-24.

[5] Abdel-Rehim M, Dahlgren M, Blomberg L. Quantification of ropivacaine and its major metabolites in human urine samples utilizing microextraction in a packed syringe automated with liquid chromatography-tandem mass spectrometry (MEPS-LC MS/MS). J Sep Sci 2006; 29: 1658-61.
[6] Abdel-Rehim M. New trend in sample preparation: on-line microextraction syringe for liquid and gas chromatography applications: I. Determination of local anaesthetics in human plasma samples using gas chromatography-mass spectrometry. J Chromatogr B 2004; 81: 317-21.

[7] Moore MJ. Clinical pharmacokinetics of cyclophosphamide. Clin Pharmacokinet 1991; 20: 194

[8] Binotto G, Trentin L, Semenzato G. Ifosfamide and cyclophosphamide: effects on immunosurveillance. Oncology 2003; 65: 17 .

[9] Sadagopan N, Cohen L, Roberts B, Collard W, Omer C. Liquid chromatography-tandem mass spectrometric quantitation of cyclophosphamide and its hydroxy metabolite in plasma and tissue for determination of tissue distribution. J Chromatogr B 2001; 759: 277-28.

[10] De Jonge ME, Van Dam SM, Hillebrand MJX, et al. Simultaneous quantification of cyclophosphamide, 4 hydroxycyclophosphamide, $\mathrm{N}, \mathrm{N}, \mathrm{N}-$ triethylenethiophosphoramide(thiotepa) and N,N,Ntriethylenephosphoramide (tepa) in human plasma byhighperformance liquid chromatography coupled with electrospray ionization tandem mass spectrometry. J Mass Spectrum 2004; 39: 262-71.

[11] Barbieri A, Sabatini L, Indiveri P, Bonfiglioli R, Lodi V, Violante FS. Simultaneous determination of low levels of methotrexate and cyclophosphamide in human urine by micro liquid chromatography/electrospray ionization tandem mass spectrometry. Rapid Commun Mass Spectrom 2006; 20: 1889-93.

[12] Silvertand LHH, Vazvaei F, Weigl P, et al. Simultaneous quantification of fludarabine and cyclophosphamide in human plasma by high performance liquid chromatography coupled with electrospray ionization tandem mass spectrometry. Rapid Commun Mass Spectrum 2005; 19: 3673-80. 